PROCEEDINGS OF THE

AMERICAN MATHEMATICAL SOCIETY

Volume 129, Number 2, Pages 397-405

S 0002-9939(00)05524-6

Article electronically published on August 29, 2000

\title{
A PROJECTION THEOREM AND TANGENTIAL BOUNDARY BEHAVIOR OF POTENTIALS
}

\author{
KOHUR GOWRISANKARAN AND DAVID SINGMAN
}

(Communicated by Albert Baernstein II)

\begin{abstract}
Let $L_{k}$ be the Weinstein operator on the half space, $\mathbb{R}_{+}^{n}$. Suppose there is a sequence of Borel sets $A_{j} \subset \mathbb{R}_{+}^{n}$ such that a certain tangential projection of $A_{j}$ onto $\mathbb{R}^{n-1}$ forms a pairwise disjoint subset of the boundary. Let $\nu$ be a finite test measure on the boundary for a specific non-isotropic Hausdorff measure. The measure $\nu$ is carried back to a measure $\lambda$ on a subset of $\bigcup A_{j}$ by the projection. We give an upper bound for the Weinstein potential corresponding to the measure $d \lambda / x_{n}$ in terms of a universal constant and a Weinstein subharmonic function. We use this upper bound to deduce a result concerning tangential behavior of Weinstein potentials at the boundary with the exception of sets on the boundary of vanishing non-isotropic Hausdorff measure.
\end{abstract}

\section{IntRoduction AND STATEMENT OF RESUlts}

Let $\mathbb{R}_{+}^{n}=\left\{x=\left(x_{1}, \ldots, x_{n}\right)=\left(x^{\prime}, x_{n}\right) \in \mathbb{R}^{n}: x_{n}>0\right\}$ denote the upper halfspace in $\mathbb{R}^{n}, n \geq 2$. The boundary of this set is $\mathbb{R}^{n-1}$. It is a well-known result due to Privalov $[\mathrm{P}]$ that the classical Green's potentials on $\mathbb{R}_{+}^{n}$ have perpendicular limit zero for Lebesgue almost every boundary point in $\mathbb{R}^{n-1}$. One way to prove this is to make use of maximal function techniques. J.L. Doob introduced a different approach in his proof of the Privalov theorem ([D], pp. 223-225). His aim was to show that the theorem follows as a consequence of the general minimal fine theory which asserts in particular that potentials have minimal fine limit zero at Lebesgue almost every boundary point. The minimal fine theory is available in quite general harmonic spaces. The key ingredient in his proof of the Privalov theorem is the following "projection theorem":

Theorem A. Let $\left\{c^{j}\right\}$ be a sequence of positive real numbers. Let, for each $j, A_{j}$ be a Borel subset of the horizontal slab $\left\{x \in \mathbb{R}_{+}^{n}: x_{n}=c^{j}\right\}$ with the property that the orthogonal projections onto $\mathbb{R}^{n-1}$ of the $A_{j}$ 's form a pairwise disjoint sequence of subsets of $\mathbb{R}^{n-1}$. Let $\lambda$ be the measure on $\bigcup A_{j}$ induced by the Lebesgue measure on $\mathbb{R}^{n-1}$. Then there exists a constant $c$ independent of the choice of $\left\{c^{j}\right\}$ and the

Received by the editors August 27, 1998 and, in revised form, April 9, 1999.

2000 Mathematics Subject Classification. Primary 31B25.

Key words and phrases. Weinstein equation, Littlewood theorem, Weinstein potential, nonisotropic Hausdorff measure, boundary behavior, minimal fine limit.

(C)2000 American Mathematical Society 
sets $A_{j}$ such that for all $x \in \mathbb{R}_{+}^{n}$,

$$
\int \frac{G(x, y)}{y_{n}} d \lambda(y) \leq c,
$$

where $G$ is the Green's function for the Laplacian on $\mathbb{R}_{+}^{n}$.

The theorem is quite natural if one views the integrand as being an approximation to the Poisson kernel, and so it is plausible that the integral is bounded by a multiple of the harmonic measure at $x$ of the orthogonal projection of the union of the $A_{j}$ 's. Since the projections are pairwise disjoint, this harmonic measure is at most 1 .

T.J. Lyons et al. LMT made use of Doob's method in their proof of a Privalov type theorem for potentials on a rank 1 symmetric space. Their proof also involves a projection theorem and the minimal fine theory. However, in the literature, the technique has not as yet been used to deduce tangential boundary behavior results for potentials. Recently we proved a minimal fine limit result for a class of potentials on a halfspace such that the associated exceptional set is of a certain Hausdorff measure zero rather than Lebesgue measure zero (GS2]). It is our aim in this paper to show that Doob's technique can be adapted so that we can deduce tangential behavior results for this class of potentials as a consequence of our minimal fine theorem. Of course the key step is a suitable projection theorem (Theorem 1 below).

The differences between our theorem and Doob's can be outlined as follows: whereas he was interested in perpendicular limits, our interest in tangential limits implies our $A_{j}$ 's have pairwise disjoint projections on the boundary with respect to a certain tangential projection; whereas his exceptional sets turned out to be of Lebesgue measure zero while ours are of Hausdorff measure zero, the measure $\lambda$ which we place on $\bigcup A_{j}$ is induced by a test measure $\nu$ for Hausdorff measure on $\mathbb{R}^{n-1}$; whereas the potential defined in $(\mathbb{1})$ was bounded by a multiple of 1 (which is the Poisson integral of the Lebesgue measure), the potential we construct in Theorem 1 (defined in (2) below) is bounded by a multiple of a function related to the Poisson integral of $\nu$. Again, Theorem 1 is plausible, since the integrand of the potential we construct is an approximation to the Poisson kernel, and so we expect the integral to be bounded by a multiple of the $\nu$-harmonic measure at $x$ of the tangential projections of the $A_{j}$ 's. Since these projections are disjoint, the latter is bounded by the Poisson integral of $\nu$ evaluated at $x$.

We shall prove our results in the more general setting of Weinstein harmonic spaces. More precisely, for $k \in \mathbb{R}$, the Weinstein equation with parameter $k$ is $L_{k}(f)=0$ where

$$
L_{k}(f)=\sum_{j=1}^{n} \frac{\partial^{2} f}{\partial x_{j}^{2}}+\frac{k}{x_{n}} \frac{\partial f}{\partial x_{n}}=0 .
$$

The $C^{2}$ functions that satisfy the Weinstein equation form a strong Brelot harmonic space $\mathrm{He}$. We shall use terms such as harmonic functions, potentials, reduced functions, domination principle, etc. referring to the potential theoretical concepts corresponding to these harmonic spaces. There is a correspondence principle which associates all of the potential theoretical concepts and formulae of the case $k<1$ to that of $k>1$ (GS1], page 636). Using this principle, it is easy to deduce results in the case $k>1$ from analogous results for the case $k<1$. In this paper we shall restrict ourselves to the case $k<1$. 
We now recall ([GS1], Proposition 1 ) that the potential with pole at $y$ is the function

$$
G_{k}(x, y)=a_{n, k} x_{n}^{1-k} y_{n} \int_{0}^{\pi} \frac{\sin ^{1-k} t}{\left[|x-y|^{2}+2 x_{n} y_{n}(1-\cos t)\right]^{(n-k) / 2}} d t,
$$

where

$$
a_{n, k}=\frac{\Gamma\left(\frac{n-k}{2}\right)}{2 \pi^{n / 2} \Gamma\left(\frac{2-k}{2}\right)} \quad \text { for } k \leq 1
$$

Let $\mu$ be a measure (we use the term measure to indicate a nonnegative Radon measure) on $\mathbb{R}_{+}^{n}$. The function $G_{k} \mu(x)=\int G_{k}(x, y) d \mu(y)$ is a potential (i.e. $G_{k} \mu$ not identically $\infty)$ on $\mathbb{R}_{+}^{n}$ if $\mu$ verifies the condition $\int_{\mathbb{R}_{+}^{n}} \frac{y_{n}}{(1+|y|)^{n-k}} d \mu(y)<\infty$ (GS1, Proposition 2).

Let $\tau \geq 1$ and let us define on $\mathbb{R}^{n-1}$ the pseudodistance

$$
d^{\tau}(x, y)=\sqrt{\left(x_{1}-y_{1}\right)^{2 \tau}+\sum_{j=2}^{n-1}\left(x_{j}-y_{j}\right)^{2}}
$$

Let $B^{\tau}(x, r)=\left\{y \in \mathbb{R}^{n-1}: d^{\tau}(x, y)<r\right\}$ denote the $d^{\tau}$-open balls. We also define the $P^{\tau}$-projection of $x=\left(x_{1}, \ldots, x_{n}\right)$ in $\mathbb{R}_{+}^{n}$ to $\mathbb{R}^{n-1}$ by

$$
P^{\tau}(x)=\left(x_{1}-x_{n}^{1 / \tau}, x_{2}, \ldots, x_{n-1}\right) .
$$

Suppose $\Gamma_{0}^{\tau}$ is the graph of the curve in the $\left(x_{1}, x_{n}\right)$-plane through the origin and of degree of tangency $\tau$ given by $\Gamma_{0}^{\tau}=\left\{\left(t, 0, \ldots, 0, t^{\tau}\right): t \geq 0\right\}$. For each $x \in \mathbb{R}_{+}^{n}$ consider the tangential path which connects $x$ to $P^{\tau}(x)$ whose graph is $\Gamma_{P^{\tau}(x)}^{\tau}=$ $\left(P^{\tau}(x), 0\right)+\Gamma_{0}^{\tau}$. Note that $P^{\tau}$-projection carries any point along this path to the "base point" $P^{\tau}(x)$. We shall refer to the limiting behavior of functions along these curves as $\Gamma^{\tau}$ limiting behavior.

Theorem 1. Let $0<\omega \leq 1$ and $1 \leq \tau \leq 1 / \omega$. Let $\nu$ be a measure on $\mathbb{R}^{n-1}$ such that for every non-isotropic ball $B^{\tau}(x, r)$ with $r>0$, the measure verifies $\nu\left(B^{\tau}(x, r)\right) \leq r^{n-2+\omega}$. Let $\left\{x^{j}\right\}$ be a sequence of points in $\mathbb{R}_{+}^{n}$. Let, for each $j$, $A_{j}$ be a Borel subset of $\left\{x \in \mathbb{R}_{+}^{n}: x_{1}=x_{1}^{j}\right\}$ with the property that $\left\{P^{\tau}\left(A_{j}\right)\right\}_{j}$ is a pairwise disjoint sequence of subsets of $\mathbb{R}^{n-1}$. Let $\lambda$ be the measure on $\bigcup A_{j}$ which is the inverse image of $\nu$ under $P^{\tau}$. (Note: $P^{\tau}$ is one-to-one and bi-continuous on $\left.\cup A_{j}.\right)$ Then there exists a constant $c$ independent of the choice of $x^{j}$ and the sets $A^{j}$ such that for all $x \in \mathbb{R}_{+}^{n}$,

$$
\int \frac{G_{k}(x, y)}{y_{n}} d \lambda(y) \leq \frac{c}{x_{n}^{1-\omega}} .
$$

Let us recall the definition of the non-isotropic Hausdorff measure $H_{\alpha}^{\tau}$ of subsets of $\mathbb{R}^{n-1}$ corresponding to $\tau \geq 1$ and $\alpha>0$ [GS1]:

$$
H_{\alpha}^{\tau}(F)=\sup _{\epsilon>0}\left[\inf \left\{\sum_{j=1}^{\infty} r_{j}^{\alpha}: \forall j, r_{j}<\epsilon, \exists x_{j} \text { such that } F \subset \bigcup_{j} B^{\tau}\left(x_{j}, r_{j}\right)\right\}\right] \text {. }
$$

It is easily seen that when $\tau=1, H_{\alpha}^{1}$ is the usual $\alpha$-dimensional Hausdorff measure. We also note that when $\alpha=n-2+\frac{1}{\tau}$, the corresponding $H_{\alpha}^{\tau}$ measure is a multiple of the Lebesgue measure on $\mathbb{R}^{n-1}$. We recall 
Theorem B (GS1], Theorem 1). Let $\tau \geq 1$ and $0<\alpha \leq n-2+\frac{1}{\tau}$. Let $K$ be a compact subset of $\mathbb{R}^{n-1}$. Then $H_{\alpha}^{\tau}(K)>0$ if and only if there exists a nontrivial measure $\sigma$ supported by $K$ such that for every $x \in \mathbb{R}^{n-1}$ and every $r>0$, $\sigma\left(B^{\tau}(x, r)\right) \leq r^{\alpha}$

Let us define the $\tau$-radius $r_{\tau}(E)=r(E)$ of a set $E \subset \mathbb{R}^{n-1}$ to be the infimum of the set of all $r>0$ such that there exists an $x \in \mathbb{R}^{n-1}$ with $E \subset \overline{B^{\tau}(x, r)}$. Let us further introduce the following five set functions. For each $\delta>0$ let

$$
\begin{aligned}
& \omega_{\delta}(F)=\inf \left\{\sum r_{j}^{\alpha}: r_{j}<\delta, \exists x_{j} \in \mathbb{R}^{n-1} \text { with } F \subset \bigcup_{j} B^{\tau}\left(x_{j}, r_{j}\right)\right\}, \\
& \sigma_{\delta}(F)=\inf \left\{\sum\left(r\left(S_{j}\right)\right)^{\alpha}: r\left(S_{j}\right)<\delta, \exists S_{j} \subset \mathbb{R}^{n-1} \text { with } F \subset \bigcup_{j} S_{j}\right\} .
\end{aligned}
$$

Define $\mu_{\delta}$ (respectively $\nu_{\delta}$ ) to be the number obtained as in $\sigma_{\delta}(F)$ by restricting the sets $S_{j}$ to be open (respectively closed). Finally, we get $\tau_{\delta}(F)$ as in $\sigma_{\delta}(F)$ except we require the stricter condition that $\bigcup_{j} S_{j}=F$. The following lemma is proved in a routine way.

Lemma. Let $0<\delta<\epsilon, F \subset \mathbb{R}^{n-1}$. Then $\mu_{\epsilon}(F) \leq \omega_{\epsilon}(F) \leq \nu_{\delta}(F)=\sigma_{\delta}(F)=$ $\tau_{\delta}(F) \leq \mu_{\delta}(F)$. Hence when $\epsilon$ decreases to 0 , the supremum (limit) of each of the numbers $\mu_{\epsilon}(F), \omega_{\epsilon}(F), \nu_{\epsilon}(F), \sigma_{\epsilon}(F)$, and $\tau_{\epsilon}(F)$ is exactly $H_{\alpha}^{\tau}(F)$ introduced above.

Theorem 2. If $F$ is any Borel set contained in $\mathbb{R}^{n-1}$ with $H_{\alpha}^{\tau}(F)>\lambda>0$ for some real number $\lambda$, then there exists a compact set $K \subset F$ such that $H_{\alpha}^{\tau}(K)>\lambda$.

The proof of the above result is far from routine or easy. It follows exactly on the same lines as that of the rather difficult proof of inner regularity of Hausdorff measures defined using a metric as found in $[\mathrm{R}]$. The fact that $\tau$ is a pseudodistance and not a metric does not create any substantial difficulties. The result is proved by adapting the proof of the case of the metric space $([\mathrm{R}]$, pages $26-39,44-52,84-$ 99). The adaptation of the proofs in that book to our case of pseudodistance is facilitated by the above lemma.

The next result was proved in [GS1] using techniques of maximal functions. Here we deduce the result as a consequence of the projection result, Theorem 1.

Theorem 3. Let $0<\omega \leq 1$ and $1 \leq \tau \leq 1 / \omega$. Let $\mu$ be a measure on $\mathbb{R}_{+}^{n}$ for which $G_{k} \mu$ is a potential. Let, in addition, $\mu$ satisfy the growth condition $\int_{F} y_{n}^{\omega} d \mu<\infty$ for all Borel sets $F \subset \mathbb{R}_{+}^{n}$ such that $F$ is bounded in $\mathbb{R}^{n}$. Then, for all $x \in \mathbb{R}^{n-1}$ except for $x$ in a set $F$ such that $H_{n-2+\omega}^{\tau}(F)=0$, the $\Gamma_{x}^{\tau}$-limit of $G_{k} \mu=0$.

\section{Proof of Theorem 1}

Let us first briefly recall some notations and results from [GS1]. Accordingly, we denote the Euclidean ball in $\mathbb{R}_{+}^{n}$ of radius $r$ and center $x$ by $B(x, r)$ and the non-isotropic (i.e. $d^{\tau}$ ) ball in $\mathbb{R}^{n-1}$ centered at $y$ and of radius $r$ by $B^{\tau}(y, r)$. We also have the following inclusion for $x \in \mathbb{R}_{+}^{n}$ and $r>0$ :

$$
P^{\tau}(B(x, r)) \subset B^{\tau}\left(P^{\tau}(x), 2^{\left(\tau-\frac{1}{2}\right)} r\right) .
$$


We claim that for all $x \in \mathbb{R}_{+}^{n}$ and $r>0$, the measure $\lambda$ verifies the following inequality:

$$
\lambda(B(x, r)) \leq c \cdot r^{n-2+\omega} .
$$

Here and in what follows, we shall use the letter $c$ to represent a quantity that may vary from line to line but does not depend in an important way on the parameters of interest.

From the definition of the measure $\lambda$ and our disjointness assumption on $\left\{P^{\tau}\left(A_{j}\right)\right\}_{j}$, we have

$$
\begin{aligned}
\lambda(B(x, r)) & =\sum_{j} \lambda\left[B(x, r) \cap A_{j}\right] \\
& =\sum_{j} \nu\left(P^{\tau}\left[B(x, r) \cap A_{j}\right]\right) \\
& \leq \sum_{j} \nu\left[P^{\tau}(B(x, r)) \cap P^{\tau}\left(A_{j}\right)\right] \\
& =\nu\left[P^{\tau}(B(x, r)) \cap\left(\bigcup_{j} P^{\tau}\left(A_{j}\right)\right)\right] \\
& \leq \nu\left[P^{\tau}(B(x, r))\right] \\
& \leq c \cdot r^{n-2+\omega} \text { by }(3),
\end{aligned}
$$

proving the claim.

For the remainder of the proof, let us fix an $x \in \mathbb{R}_{+}^{n}$. Consider the potential $p(x)=\int G_{k}(x, y)\left(1 / y_{n}\right) d \lambda(y)=I+I I$, where $I$ and $I I$ are obtained respectively by performing the integral on the set of all $y$ where $|x-y|>x_{n} / 2$ and on the complement of that set. We first estimate $I$ using the inequality $G_{k}(x, y) \leq c x_{n}^{1-k} y_{n} /|x-y|^{n-k}$ ([GS1], page 639).

$$
\begin{aligned}
I & \leq c \int_{|x-y|>x_{n} / 2} \frac{x_{n}^{1-k}}{|x-y|^{n-k}} d \lambda(y) \\
& \leq c \sum_{m=0}^{\infty} \int_{2^{m-1} x_{n} \leq|x-y| \leq 2^{m} x_{n}} \frac{x_{n}^{1-k}}{|x-y|^{n-k}} d \lambda(y) \\
& \leq c \sum_{m=0}^{\infty} \frac{x_{n}^{1-k}}{\left(2^{m-1} x_{n}\right)^{n-k}} \lambda\left[B\left(x, 2^{m} x_{n}\right)\right] \\
& \leq c \sum_{m=0}^{\infty} \frac{x_{n}^{1-n}}{\left(2^{m}\right)^{n-k}}\left(2^{m} x_{n}\right)^{n-2+\omega} \text { from }(4) \\
& \leq \frac{c}{x_{n}^{1-\omega}} \sum_{m=0}^{\infty}\left(2^{m}\right)^{k+\omega-2} \\
& \leq \frac{c}{x_{n}^{1-\omega}}
\end{aligned}
$$

since the geometric sum above is convergent by virtue of the assumptions $k<1$ and $\omega \leq 1$. 
To estimate $I I$, we first consider the case when $n \geq 3$. In this case, we recall the following estimate for $G_{k}$ (page 639, [GS1]): $G_{k}(x, y) \leq c \frac{x_{n}^{-k / 2} y_{n}^{k / 2}}{|x-y|^{n-2}}$.

$$
\begin{aligned}
I I & =\int_{|x-y| \leq x_{n} / 2} \frac{G_{k}(x, y)}{y_{n}} d \lambda(y) \\
& \leq c \int_{|x-y| \leq x_{n} / 2} \frac{x_{n}^{-k / 2} y_{n}^{k / 2}}{y_{n}} \frac{1}{|x-y|^{n-2}} d \lambda(y) \\
& \leq c \int_{|x-y| \leq x_{n} / 2} \frac{1}{x_{n}|x-y|^{n-2}} d \lambda(y),
\end{aligned}
$$

since $2 / 3 \leq x_{n} / y_{n} \leq 2$. Hence,

$$
\begin{aligned}
I I & \leq \frac{c}{x_{n}} \sum_{m=1}^{\infty} \int_{\frac{x_{n}}{2^{m+1}} \leq|x-y| \leq \frac{x_{n}}{2^{m}}} \frac{1}{|x-y|^{n-2}} d \lambda(y) \\
& \leq \frac{c}{x_{n}} \sum_{m=1}^{\infty} \frac{2^{(m+1)(n-2)}}{x_{n}^{n-2}} \lambda\left[B\left(x, x_{n} / 2^{m}\right)\right] \\
& \leq \frac{c}{x_{n}^{n-1}} \sum_{m=1}^{\infty} 2^{m(n-2)}\left(\frac{x_{n}}{2^{m}}\right)^{n-2+\omega} \\
& =\frac{c}{x_{n}^{1-\omega}} \sum_{m=1}^{\infty} 2^{-m \omega} \\
& \leq \frac{c}{x_{n}^{1-\omega}} .
\end{aligned}
$$

This completes the proof in the case $n \geq 3$.

Now consider the case $n=2$. For this we have the following estimate for the Green function (page 639, [GS1]): $G_{k}(x, y) \leq c x_{2}^{-k / 2} y_{2}^{k / 2}\left[1+\left|\log \frac{\sqrt{x_{2} y_{2}}}{|x-y|}\right|\right]$. In this case the contribution from the second integral satisfies

$$
\begin{aligned}
I I & =\int_{|x-y| \leq x_{n} / 2} G_{k}(x, y) \frac{1}{y_{2}} d \lambda(y) \\
& \leq c \int_{|x-y| \leq x_{n} / 2} \frac{x_{2}^{-k / 2}}{y_{2}^{1-k / 2}}\left[1+\left|\log \frac{\sqrt{x_{2} y_{2}}}{|x-y|}\right|\right] d \lambda(y)
\end{aligned}
$$

and as before,

$$
\begin{aligned}
& \leq \frac{c}{x_{2}} \sum_{m=1}^{\infty}\left[1+\log \left(2^{m+1}\right)\right] \lambda\left[B\left(x, x_{2} / 2^{m}\right)\right] \\
& \leq \frac{c}{x_{2}} \sum_{m=1}^{\infty}[1+(m+1) \log 2]\left(x_{2} / 2^{m}\right)^{\omega} \\
& =\frac{c}{x_{2}^{1-\omega}}\left[\sum_{m=1}^{\infty} 2^{-m \omega}+\log 2 \sum_{m=1}^{\infty}(m+1) 2^{-m \omega}\right] \\
& \leq \frac{c}{x_{2}^{1-\omega}} .
\end{aligned}
$$

This concludes the proof of Theorem 1 . 


\section{Proof of Theorem 3}

Let $\mu$ be a measure on $\mathbb{R}_{+}^{n}$ verifying the growth condition stated in Theorem 3 Let $G \mu$ be the potential corresponding to $\mu$. Let $E=\{x: G \mu(x)>1\}$ and let $E_{m}$ be the set $E \cap\left\{x: x_{n}<1 / m\right\}$. Note that the set $F$ of $P^{\tau}$-limit points of $E$ consists precisely of those $x^{\prime} \in \mathbb{R}^{n-1}$ such that $\left(P^{\tau}\right)^{-1}\left(\left\{x^{\prime}\right\}\right) \cap E_{m} \neq \emptyset$ for all $m \geq 1$. $F$ is also obtained by taking the intersection over $m$ of the $P^{\tau}$ image of $E_{m}$. Since $P^{\tau}$ is an open map, $F$ is a $G_{\delta}$ set and therefore Borel. To prove the theorem, it suffices by Theorem 2 to show that $H_{n-2+\omega}^{\tau}(K)=0$ for arbitrary compact subsets $K$ of $F$. To do this, we have to show that $\nu(K)=0$ for every measure $\nu$ supported by $K$ and verifying the inequality $\nu\left(B^{\tau}\left(x^{\prime}, r\right)\right) \leq r^{n-2+\omega}$ for all $x^{\prime} \in \mathbb{R}^{n-1}$ and all $r>0$ (Theorem 1, GS1). Accordingly, let us fix such a measure $\nu$ on a fixed compact subset $K \subset F$.

For each $x \in E_{m}$, we associate two positive numbers $s(x)$ and $t(x), 0<t(x)<$ $s(x)$, to satisfy the specifications below. In view of the easily verified fact

$$
\lim _{s \rightarrow 0^{+}} \frac{\left(x_{n}+s\right)^{1 / \tau}-\left(x_{n}-s\right)^{1 / \tau}}{2 s}=\frac{1}{\tau x_{n}^{(\tau-1) / \tau}},
$$

we can choose $s(x)>0$ such that for all $t$ satisfying $0<t<s(x)$, the following inequality is valid:

$$
\frac{1}{\tau} \frac{t}{x_{n}^{(\tau-1) / \tau}} \leq\left(x_{n}+t\right)^{1 / \tau}-\left(x_{n}-t\right)^{1 / \tau}<\frac{3}{\tau} \frac{t}{x_{n}^{(\tau-1) / \tau}} .
$$

Let $t(x)=x_{n}^{(\tau-1) / \tau} s(x)$. We further assume that $s(x)$ is chosen sufficiently small so that the following rectangular set

$R(x)=\left\{\left(y_{1}, \ldots, y_{n}\right): y_{1}=x_{1},\left|x_{j}-y_{j}\right| \leq s(x) \forall j=2, \ldots n-1,\left|y_{n}-x_{n}\right| \leq t(x)\right\}$

is contained in $E_{m}$. We observe that

$$
P^{\tau}[R(x)]=\left\{\left(x_{1}-y_{n}^{1 / \tau}, y_{2}, \ldots, y_{n-1}\right): y \in R(x)\right\} .
$$

This is a closed rectangular set in $\mathbb{R}^{n-1}$ and the length of its sides in the $y_{2}, \ldots, y_{n-1}$ variables is $2 s(x)$, and in the direction of the first axis it is $\left(x_{n}+t(x)\right)^{1 / \tau}-$ $\left(x_{n}-t(x)\right)^{1 / \tau}$. This last number lies between $s(x) / \tau$ and $3 s(x) / \tau$ by (7). It follows that the ratios of the length of sides of the rectangle $P^{\tau}[R(x)]$ lies between two (non-zero) positive numbers that depend only on $\tau$. We also note that in the above choice of $s(x)$, we have the freedom to replace it with any smaller positive number.

It is simple to verify from the formula for the Green function that for all $y^{\prime} \in K$ and all $x \in \mathbb{R}_{+}^{n}$,

$$
\lim _{z \rightarrow y^{\prime}} \frac{G_{k}(x, z)}{z_{n}}=c \frac{x_{n}^{1-k}}{\left|x-y^{\prime}\right|^{n-k}}=c \mathbf{K}\left(x, y^{\prime}\right)
$$

for a constant $c$ independent of $y^{\prime} \in K$ and $x \in \mathbb{R}_{+}^{n}$, where $\mathbf{K}\left(x, y^{\prime}\right)$ is the Martin kernel with pole at $y^{\prime}$. Let us now fix $\xi \in \mathbb{R}_{+}^{n}$ and $\epsilon>0$. Using the compactness of $K$, we may find a $\delta>0$ such that for all $z \in \mathbb{R}_{+}^{n}$ with $\left|z-y^{\prime}\right|<\delta$ and $y^{\prime} \in K$, we have

$$
\left|\frac{G_{k}(\xi, z)}{z_{n}}-c \mathbf{K}\left(\xi, y^{\prime}\right)\right|<\epsilon .
$$

Now, for each $y^{\prime} \in K$, we have necessarily a point $x=x\left(y^{\prime}\right) \in E_{m}$ such that $P^{\tau}(x)=y^{\prime}$. Choose such a point to satisfy the additional condition that $\left|x-y^{\prime}\right|<$ 
$1 / 2$. Now, assign a rectangle $R^{m}(x)$ as above ensuring that $s(x)$ is chosen so small that $R^{m}(x) \subset E_{m} \cap B\left(y^{\prime}, \delta\right)$. Let $\Gamma^{m}\left(y^{\prime}, x\right)=P^{\tau}\left(R^{m}(x)\right)$. Then the mapping $y^{\prime} \rightarrow \Gamma^{m}\left(y^{\prime}, x\left(y^{\prime}\right)\right)$ of $K$ into subsets of $\mathbb{R}^{n-1}$ verifies all the conditions of the Morse covering theorem [M] as described on page 6 of $\mathrm{Guz}$ (see also [L]). We conclude that there is a number $\Theta$ depending only on the dimension $(n-1)$ with the following property: there are at most $\Theta$ subfamilies of $\left\{\Gamma^{m}\left(y^{\prime}, x\right)\right\}_{y^{\prime} \in K}$ forming a cover of $K$ with the property that each subfamily consists of a countable number of mutually disjoint sets of the above collection.

Let us take $p$ between 1 and $\Theta$ and the corresponding subfamily $\left\{\Gamma^{m, p}\left(y_{l}^{\prime}, x_{l}\right)\right\}_{l=1}^{\infty}$. Let us denote the corresponding rectangles centered at $x_{l}$ by $R_{p}^{m}\left(x_{l}\right)$. Let $\lambda_{l}^{m, p}$ be the measure on $R_{p}^{m}\left(x_{l}\right)$ which is obtained by transporting $\nu$ backwards as described in the statement of Theorem 1. Let $\lambda^{m, p}=\sum_{l=1}^{\infty} \lambda_{l}^{m, p}$. (Note that for fixed $p$, the sets $R_{p}^{m}\left(x_{l}\right)$ are mutually disjoint.) By Theorem 1 , there is a constant $c$ independent of $m$ and $p$ such that

$$
q_{m, p}(x)=\int \frac{G_{k}(x, y)}{y_{n}} d \lambda^{m, p}(y) \leq \frac{c}{x_{n}^{1-\omega}} .
$$

This potential $q_{m, p}$ is locally bounded because of (9) and further its support is contained in $E_{m}$. We conclude by using the Domination Principle (Axiom D) that

$$
q_{m, p}(x) \leq c R_{x_{n}^{\omega-1}}^{E} \text { for all } x \in \mathbb{R}_{+}^{n} .
$$

Also, the method of construction of the measure $\lambda^{m, p}$ shows that

$$
\begin{aligned}
q_{m, p}(x) & =\int \frac{G_{k}(x, z)}{z_{n}} d \lambda^{m, p}(z) \\
& =\int_{A_{p}} \phi_{m, p}\left(y^{\prime}\right) d \nu\left(y^{\prime}\right),
\end{aligned}
$$

where $A_{p}$ is the disjoint union $\bigcup_{l} \Gamma^{m, p}\left(y_{l}^{\prime}, x_{l}\right)$ and for $y^{\prime} \in \Gamma^{m, p}\left(y_{l}^{\prime}, x_{l}\right), \phi_{m, p}\left(y^{\prime}\right)=$ $G_{k}(x, z) / z_{n}$ with $z=\left(P^{\tau}\right)^{-1}\left(y^{\prime}\right) \cap R^{m, p}\left(x_{l}\right)$. Hence, from inequality (8) we deduce that

$$
q_{m, p}(\xi) \geq c \int_{A_{p} \cap K}\left[\mathbf{K}\left(\xi, y^{\prime}\right)-\epsilon\right] d \nu\left(y^{\prime}\right) .
$$

Hence, for this $\xi$,

$$
q_{m, p}(\xi) \geq c \int_{A_{p} \cap K} \mathbf{K}\left(\xi, y^{\prime}\right) d \nu\left(y^{\prime}\right)-\epsilon\|\nu\| .
$$

Using the finite subadditivity of measures we conclude that

$$
\sum_{p} q_{m, p}(\xi) \geq c \int_{K} \mathbf{K}\left(\xi, y^{\prime}\right) d \nu\left(y^{\prime}\right)-\epsilon\|\nu\| .
$$

We now combine inequalities (10) and (11) and note that we can let $\epsilon \rightarrow 0$ to get the inequality

$$
R_{x_{n}^{\omega-1}}^{E}(\xi) \geq c \int_{K} \mathbf{K}\left(\xi, y^{\prime}\right) d \nu\left(y^{\prime}\right)
$$

Evidently, this inequality is true for all $\xi \in \mathbb{R}_{+}^{n}$. We note that the function on the right side of (12) is a positive $L_{k}$-harmonic function with canonical Martin representing measure $c \nu$ BCB1]. Also, it is proved in the proof of Theorem 1 in 
[GS2] that the function $R_{x_{n}^{\omega-1}}^{E}$ is an $L_{k}$-potential. It follows that the function on the right side of the inequality (12) is identically zero; hence $\nu \equiv 0$. This concludes the proof.

\section{REFERENCES}

[BCB1] B. Brelot-Collin and M. Brelot, Représentation intégrale des solutions positives de l'equation $L_{k}(f)=\sum_{i=1}^{n} \frac{\partial^{2} f}{\partial x_{i}^{2}}+\frac{k}{x_{n}} \frac{\partial f}{\partial x_{n}}=0,(\mathrm{k}$ constante réele) dans le demi-espace $\mathrm{E}\left(x_{n}>0\right)$, de $\mathbb{R}^{n}$, Bull. Acd. Royale de Belg. 58 (1972), 317-326. MR 47:7052

[BCB2] B. Brelot-Collin and M. Brelot, Allure à la frontière des solutions positives de l'equation de Weinstein $L_{k}(u)=\Delta u+\frac{k}{x_{n}} \frac{\partial u}{\partial x_{n}}=0$ dans le demi-espace $\mathrm{E}\left(x_{n}>0\right)$ de $\mathbb{R}^{n}(n \geq 2)$, Bull Acad. Royale de Belg. 59 (1973), 1100-1117. MR 50:2528

[D] J. L. Doob, Classical potential theory and its probabilistic counterpart, Springer-Verlag, 1984. MR 85k:31001

[GS1] Kohur GowriSankaran and David Singman, A generalized Littlewood theorem for Weinstein potentials on a halfspace, Ill. J. Math., 41 no. 4 (1997), 630-647. MR 98m:31007

[GS2] Kohur GowriSankaran and David Singman, Minimal fine limits for a class of potentials, to appear, Potential Analysis.

[Guz] Miguel de Guzmán, Differentiation of Integrals in $\mathbb{R}^{n}$, Lecture Notes in Math. \#481, Springer-Verlag. MR 80b:28005

[H] W. K. Hayman, Subharmonic Functions, Volume 2, London Math. Society Monograph No. 20, Academic Press, 1989. MR 91f:31001

[HK] W. K. Hayman and P. B. Kennedy, Subharmonic Functions, Volume 1, Academic Press, 1976. MR 57:665

[He] Mme. R.-M. Hervé, Recherches axiomatiques sur la théorie des fonctions surharmoniques et du potentiel, Ann. Inst. Fourier t. 12 (1962), 415-571. MR 25:3186

$[\mathrm{K}] \quad$ Erwin Kreyszig, Advanced Engineering Mathematics, seventh edition, Wiley, Appendix 3. CMP 93:03

[L] Peter A. Loeb, Opening covering theorems of Besicovitch and Morse, to appear.

[LMT] T. J. Lyons, K. B. Macgibbon, J. C. Taylor, Projection theorems for hitting probabilities and a theorem of Littlewood, J. Funct. Anal. 59(1984), no. 3 470-489. MR 86c:31002

[M] A. P. Morse, Perfect blankets, Trans. Amer. Math. Soc. 6 1947, 418-442. MR 8:571h

[P] I. I. Privalov, Sur un probleme limite des fonctions sous-harmoniques, Rec. Math. (Mat. Sbornik) N. S. 41 (1934), 3-10.

[R] C. A. Rogers, Hausdorff Measures, Cambridge University Press, 1970. MR 43:7576

[St] Elias M. Stein, Singular integrals and differentiability properties of functions, Princeton University Press, 1970. MR 44:7280

[W] A. Weinstein, Generalized axially symmetric potential theory, Bull. Am. Math. Soc. 69 (1953), 20-38. MR 14:749c

Department of Mathematics, McGill University, Montreal, Quebec, Canada H3A $2 \mathrm{~K} 6$

E-mail address: gowri@math.mcgill.ca

Department of Mathematics, George Mason University, Fairfax, Virginia 22030

E-mail address: dsingman@osf1.gmu.edu 